\title{
A Universal Abstract Model for Future Movements of Moving Objects
}

\author{
Reasey Praing and Markus Schneider * \\ University of Florida \\ Department of Computer \& Information Science \& Engineering \\ Gainesville, FL 32611, USA \\ \{rpraing,mschneid\}@cise.ufl.edu
}

Summary. Recently, with the advances in mobile technology and emerging mobile applications, moving objects databases have become the focus of much research. A number of models have been proposed to handle the continuously changing geometries and positions of moving objects. While most of these models focus on managing the historical and current motion of moving objects, only a few of them have been proposed to deal with their future movements. Due to the complexity of handling the uncertainty aspect associated with future movements, these models are often restricted to a specific type of constrained movement and tailored to a certain application domain by integrating specific prediction techniques. These predictions are often based only on the past and current movements and neglect external domainspecific information which may significantly affect the future movements of moving objects. In this paper, we emphasize the separation of moving object models and prediction methods and propose a universal abstract model for future movements of moving objects. This abstract model serves as a conceptual basis for all future movement models as well as poses object requirements for the output of all prediction methods.

\section{Introduction}

Research in moving objects database technology has become increasingly important and popular due to recent advances in the supporting technologies within the fields of mobile computing, wireless communications, sensor networks, and location services. A number of work has been done to model moving objects especially in regard to their past and current movements and provide support for querying this information. Although, it is important to be able to answer queries regarding the past and current movements, it is also important and interesting, in many cases, to be able to answer queries regarding

\footnotetext{
* This work was partially supported by the National Science Foundation under grant number NSF-CAREER-IIS-0347574.
} 
the future movements of moving objects. For example, the future development of a hurricane would be one of the most critical information for emergency management agencies. Similar types of information have been found to be of significant importance in many problem areas such as collision detection or prevention in traffic control, future location service in navigation systems, and disaster relief effort in emergency management.

A few specialized models have been proposed to manage and support queries regarding future movements of moving objects. These models are often tailored to specific application domains due to their partially close dependency on specific, hard-wired prediction methods. More often than not, each of these models anticipates future object movements through certain assumptions on the objects' inertia, trajectories, and environmental or contextual constraints. Their prediction methods are almost always entirely based on the past and current movements of the objects, thus, neglecting external factors or domain-specific parameters which may significantly affect the future movements of moving objects. For instance, information such as atmospheric pressures, temperature zones, wind and ocean currents plays a major role in predicting the future evolution of a hurricane. This requires highly specialized and sophisticated prediction models and algorithms beyond those in which only the past and current object movements are considered as system parameters. In fact, the development effort for such prediction methods is a whole discipline by itself, and this task belongs to the domain experts, in this case meteorologists.

In general, we do not see it as the task of a database system to predict the future movements of a moving object. Instead, a moving objects database is to provide modeling and persistence support for storing predicted data and offer querying capability for retrieving such data. Hence, it is sufficient and reasonable to emphasize the separation between moving object models and prediction models with respect to future evolutions of moving objects. Since this separation has not been realized so far in existing future movement models, each of these models has only dealt with a specific problem area or object motion while neglecting the problem of how a moving object database can model the future movements of moving objects in general.

In this paper, we formally define and propose a universal abstract model for Future Movements of Moving Objects (FuMMO). This model is called abstract since it uses the infinite point-set concept of point-set theory to define future movements of moving objects such as moving points (mpoint), moving lines (mline), and moving regions (mregion) taking into account future evolutional properties such as uncertainty and dimensional restrictions. The model also provides a set of operations as well as querying support capability for object accesses and manipulations. The main contribution of this model is a formal conceptual basis and a firm foundation for the development of finite representation models as well as implementation models for future developments of moving objects. 
The paper is structured as follows. In Section 2, we discuss currently existing models and approaches as they are relevant in our context. We define the abstract model in Section 3. Then, in Section 4, the query capability of the model is demonstrated. Finally, we give some conclusions and anticipate future work in Section 5 .

\section{Related Work}

During the past decade, we have seen several major developments of spatiotemporal data models [1]. Among these developments, moving object models have been of significant interest in both application and research directions. In the next subsection, we discuss an existing moving object model that supports the past evolution of moving objects. Then, we take a look at a number of prediction models that support specific types of near future developments of moving objects.

\subsection{A Model for Historical Movements of Moving Objects}

Some approaches have been taken to represent the (past) movement of moving objects $[2,3]$. The past development is a historical fact that is modeled as a function from time to space $[4,5]$. For an arbitrary data type $\alpha$, the corresponding moving type of $\alpha$ is a function $\tau(\alpha)$ that provides the mapping from the temporal domain to $\alpha$, i.e.,

$$
\tau(\alpha)=\text { time } \rightarrow \alpha
$$

By applying this type constructor $(\tau)$ of moving data type to spatial data types such as point, line, and region, the definition of the corresponding moving types such as moving point (mpoint), moving line (mline), and moving region (mregion) can be obtained respectively [6].

$$
\begin{aligned}
\text { mpoint } & =\tau(\text { point })=\text { time } \rightarrow \text { point } \\
\text { mline } & =\tau(\text { line })=\text { time } \rightarrow \text { line } \\
\text { mregion } & =\tau(\text { region })=\text { time } \rightarrow \text { region }
\end{aligned}
$$

With the definition of these moving types, many complex algorithms and operations are developed to harvest valuable information from objects of these types [7]. Figure 1 illustrates a moving point and a moving region. The evolution of these objects is modeled as a function from time to space. Although, these functions are partial functions, i.e., there can be time intervals for which we do not know the locations of a moving object, it is assumed that we have precise knowledge about the evolution of spatial objects in the past. Hence, only the past evolution can be modeled, and the temporal domain cannot exceed the present into the future. Due to the inherent uncertainty nature of the future, this model is not suitable for representing the future developments of moving objects. 


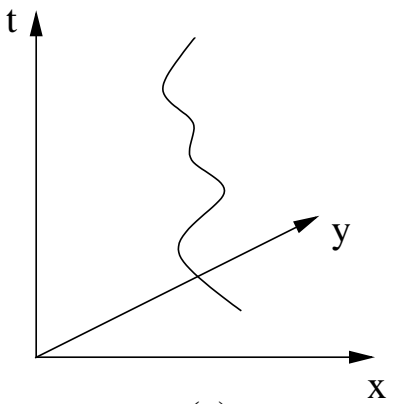

(a)

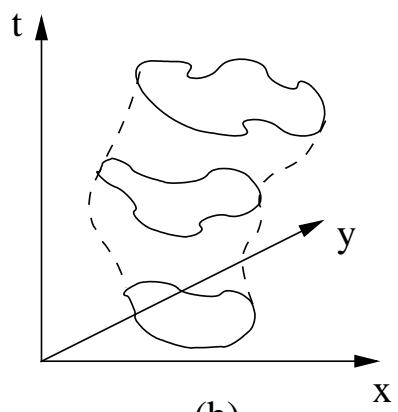

(b)

Fig. 1. A moving point (a) and a moving region (b)

\subsection{Existing Models for Future Movements of Moving Objects}

With regard to the future movements of moving objects, currently existing models are restricted to specific types of motion. The MOST model [8], which is based on the concept of motion vector, is able to represent near future developments of moving objects through an assumption on the objects' inertia that the current motion direction does not change in the near future. Although, this model is able to represent near future movement of moving object, the predictive movement is non-persistent and is rather limited to a single motion function. Future uncertainty such as probability of occurrence cannot be modeled. Even with multiple motion functions, only a finite number of possible motions can be represented.

Other models are able to capture the uncertainty aspect of the future movements through the use of a trajectory or motion plan with a constant [9] or variable uncertainty threshold [10]. Another approach presented in [11] models and predicts future movements of moving objects in a constrained network environment. An aspect all of these models have in common is that they are designed to handle a specific type of motion only; they offer or assume a specific prediction technique and provide querying capability on the predicted data.

What we see in each of these models is a vertical view that a specific restricted solution for a general problem is selected by specifying a particular prediction method and a query mechanism. However, this is not necessarily the best approach since we have shown earlier the importance of separating prediction methods from object models. Hence, we take a different approach which is a horizontal view by specifying how the future movements of moving objects can be modeled in general and independently of any prediction technique. In other words, we are interested in determining for each type of moving object, how the predicted information may look like. 


\section{FuMMO: The Abstract Model}

In this section, we present the FuMMO model by developing some concepts on how to represent the set of possible future positions or extents of moving objects in Section 3.1. Based on these concepts, we define our model data types and operations in Section 3.2.

\subsection{Representing the Future Positions and/or Extents of Moving Objects}

The future is uncertain. Thus, the central question is how the inherent uncertainty of future movements of moving objects can be handled. Most prediction models address the uncertainty issue by introducing the use of spatial or spatio-temporal ranges with associated probability to predicted data. For example, the position of the eye or the extent of a hurricane at 24 hours in the future may possibly be anywhere within a predicted region. This region is $a$ spatial range together with a specific probability distribution and represents the set of potential future positions or extents of the hurricane at 24 hours in the future. Likewise, if we are interested in the future position or extent for a certain period in the future, say from the present time to 24 hours in the future, then it can be anywhere within a predicted volume. We call this volume a spatio-temporal range. The spatial or spatio-temporal range with associated probability distribution is expected from a prediction model. In this paper, we are interested in how a spatial and spatio-temporal range can be modeled.

It is important to note that, apart from other parameters, a prediction is based on the current knowledge about a moving object up to the instant when the prediction is done. Hence, two types of prediction methods are possible here. A continuous prediction method is an approach that the future movement prediction of a moving object is conducted continuously based on the ever changing position or geometry as the object moves or evolves. A discrete prediction method, on the other hand, is an approach that a prediction is done at a specific instant of time and that multiple predictions at different instants of time are done discretely and are thus independent of one another. In this paper, we are interested in modeling the result of an individual prediction done at a specific time instant.

As mentioned earlier, we can use the concept of a spatial range to handle the inherent uncertainty of a future prediction. Therefore, we now explore all possible types of spatial ranges for each moving object type by introducing the concept of spatial range type for a moving object. We define this concept in Definition 1.

Definition 1. Let $\operatorname{dim}(\alpha)$ be a function that returns the dimension of a spatial type $\alpha$. If the evolution of a moving object of type $\tau(\alpha)$ at each time instant in the past up to the present is given by a spatial data type $\alpha$ with dimension $\operatorname{dim}(\alpha)$, then the evolution of this moving object at a time instant in the future 
can only be described by a spatial data type $\beta$ with dimension $\operatorname{dim}(\beta)$ such that $\operatorname{dim}(\alpha) \leq \operatorname{dim}(\beta) \leq n$ holds where $n$ is the dimension of the underlying space. Hence, the set of all possible spatial ranges of the moving object at a time instant in the future is represented by the spatial range type $S R(\alpha)=$ $\bigcup_{\operatorname{dim}(\alpha) \leq \operatorname{dim}(\beta) \leq n} \beta$.

Applying this definition to the moving object data types mpoint, mline, and mregion in $\mathbb{R}^{2}$, we obtain the set of spatial range types shown in Table 1 . Since the $\operatorname{dim}(\beta)$ is always greater than or equal to $\operatorname{dim}(\alpha)$, this guarantees

\begin{tabular}{|c|c|c|c|c|c|}
\hline$\tau(\alpha)$ & $\alpha$ & $\operatorname{dim}(\alpha)$ & $\operatorname{dim}(\beta)$ & $\beta$ & $S R(\alpha)$ \\
\hline mpoint & point & 0 & $\begin{array}{c}0 \\
1\end{array}$ & $\begin{array}{c}\text { point } \\
\text { line } \\
\text { region }\end{array}$ & point $\cup$ line $\cup$ region \\
\hline mline & line & 1 & $\begin{array}{c}1 \\
2\end{array}$ & $\begin{array}{c}\text { line } \\
\text { region }\end{array}$ & line $\cup$ region \\
\hline mregion & region & 2 & 2 & region & region \\
\hline
\end{tabular}

Table 1. Spatial range types corresponding to the three moving object data types

that a spatial range can always represent the set of potential future positions or extents of a moving object. In other words, a moving object can evolve or move such that, at a time instant in the future, it is a part of or equal to a spatial range without collapsing its dimension.

We can now model the set of potential positions or extents of an object by imposing a probability distribution on a spatial range. It is important to note that in our abstract model, we do not restrict the kind of distribution. This allows flexibility for any prediction model to specify the kind of distribution that is relevant to its specific application domain. We define a probabilistic spatial range (PSR) of a moving object in Definition 2.

Definition 2. The probabilistic spatial range type $P S R(\alpha)$ of a moving object of type $\tau(\alpha)$ is a pair $(S R(\alpha), f)$ such that $f$ is a probability distribution of the underlying space. For a two-dimensional space, $f$ is defined as a function $f: \mathbb{R}^{2} \rightarrow[0,1]$ such that the probability of every point in a specific spatial range of type $S R(\alpha)$ is greater than 0 and every point outside of this spatial range is 0 . Thus, $P S R(\alpha)=(S R(\alpha), f)$.

So far, we are able to model the probabilistic spatial range of a moving object only for a specific instant of time in the future. For example, a PSR can be used to model an uncertainty region in which potential future positions and/or extents of a hurricane may reside at a time instant in the future. Although this is certainly useful, one of our main objectives is to model the future movements of moving objects for a time interval in the future. Thus, by using the concept of temporal lifting mentioned earlier in Section 2, we can 
temporally lift PSR to define a probabilistic spatio-temporal range (PSTR) of a moving object as shown in Definition 3.

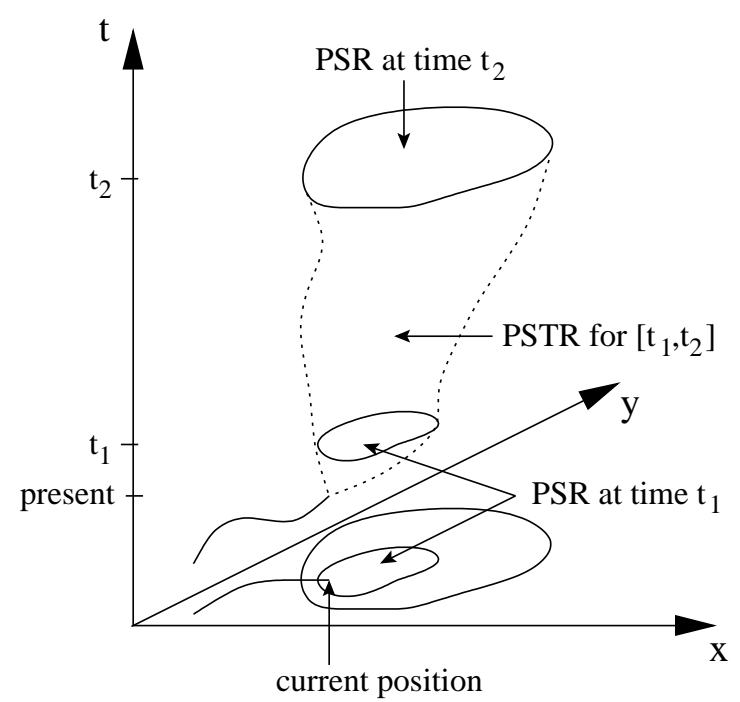

Fig. 2. Representing the future positions of a moving point using a PSTR

Definition 3. The probabilistic spatio-temporal range type $\operatorname{PSTR}(\alpha)$ of a moving object whose corresponding spatial type is $\alpha$ is defined as $\operatorname{PSTR}(\alpha)=$ $\left\{\left[t_{1}, t_{2}\right] \rightarrow P S R(\alpha) \mid\right.$ present $\left.<t_{1}<t_{2}\right\}$.

To illustrate the concepts presented, consider a moving point in an unconstrained environment. We can model each spatial range for this point using a region object. By applying a bivariate (two-dimensional) normal distribution on each spatial range, we obtain a PSR for this point. Then we can temporally lift this PSR for a specific interval in the future to obtain a corresponding PSTR. In this case, the PSTR is a three-dimensional volume in the three-dimensional spatio-temporal space and represents the set of potential future positions of the moving point. This means that the future movement trajectory of the moving point for the specified interval is predicted to be a part of the PSTR. Figure 2 illustrates an example of PSRs and a PSTR of a moving point object.

\subsection{Future Data Types and Operations}

By defining the generic types $\operatorname{PSTR}(\alpha)$ and $\operatorname{PSR}(\alpha)$ above, we can specify the corresponding spatial type $\alpha$ of a moving object type $\tau(\alpha)$ to obtain the future prediction data types as shown in Definition 4. 
Definition 4. Let fmpoint, fmline, and fmregion denote the future prediction data types for moving object types mpoint, mline, and mregion respectively. These future prediction data types are defined as:

$$
\begin{aligned}
\text { fmpoint } & =\operatorname{PSTR}(\text { point })=\left\{\left[t_{1}, t_{2}\right] \rightarrow P S R(\text { point }) \mid \text { present }<t_{1}<t_{2}\right\} \\
\text { fmline } & =\operatorname{PSTR}(\text { line })=\left\{\left[t_{1}, t_{2}\right] \rightarrow \operatorname{PSR}(\text { line }) \mid \text { present }<t_{1}<t_{2}\right\} \\
\text { fmregion } & =\operatorname{PSTR}(\text { region })=\left\{\left[t_{1}, t_{2}\right] \rightarrow P S R(\text { region }) \mid \text { present }<t_{1}<t_{2}\right\}
\end{aligned}
$$

With this definition, we are imposing object model requirements for the output of all prediction models. For example, at a time $t$ in the future, the future extent prediction of a moving region object cannot be a PSR consisting of a spatial range of type point or line. This would violate the spatial range type definition and allow a moving object to collapse its dimension.

Having modeled the future prediction of a moving object, the next question is what can we do with this model? We define a set of generic operations on the future prediction of a moving object as follows. Let $\gamma$ denote one of the future prediction data types fmpoint, fmline, and fmregion:

$$
\begin{aligned}
\text { startTime } & : \gamma \rightarrow \text { time } \\
\text { endTime } & : \gamma \rightarrow \text { time } \\
\text { psrAt } & : \text { fmpoint } \times \text { time } \rightarrow P S R(\text { point }) \\
\text { psrAt } & : \text { fmline } \times \text { time } \rightarrow P S R(\text { line }) \\
\text { psrAt } & : \text { fmregion } \times \text { time } \rightarrow P S R(\text { region }) \\
\text { spatialRange } & : P S R(\alpha) \rightarrow S R(\alpha) \\
\text { probAt } & : \gamma \times \text { point } \times \text { time } \rightarrow \text { real }
\end{aligned}
$$

The operation startTime and endTime retrieve the start and end time instants of a future prediction respectively. At a specific time instant between the start and end time, the operation psr At returns the corresponding PSR of the prediction. With this PSR, we can obtain the spatial range of the PSR by using the operation spatialRange. Finally, given a point in space we can determine the probability of this point being the future position or part of the extent of a moving object at a time instant between the start and end time by using the operation probAt operation. This operation first determines the corresponding PSR and then applies the coordinates of the given point to the probability distribution of the PSR to obtain result.

\section{Querying FuMMO}

The presented future prediction data types can be embedded into any extensible DBMS data model as attribute data types. The operations can be 
registered in the DBMS such that they are available for use in queries. For example, we can integrate these data types into the relational model and construct the following relations:

hurricanes (name:string, path:mregion, prediction:fmregion)

cities (name:string, position:point)

With these relations, we can then ask a query "Give me the start time and end time of the current prediction of hurricane Katrina":

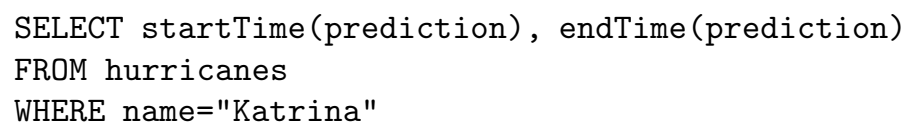

This query is only a simple retrieval of the start and end time properties of the current prediction. We can ask a more interesting question "What region will potentially be affected by hurricane Katrina 12 hours from now?"

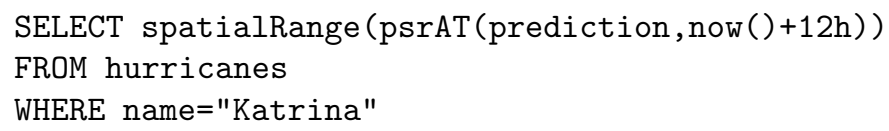

Beyond these queries, we can also ask questions relating to point probability such as "What is the probability of the city of Miami being affected by hurricane Katrina 24 hours from now?"

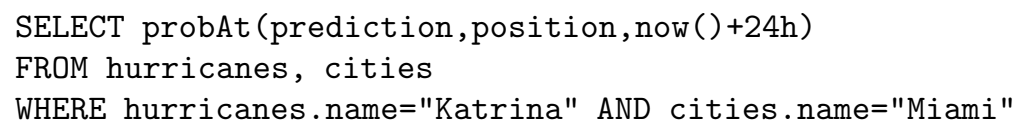

These queries illustrate the use of our operations with the future prediction of a moving object. Many other queries can be asked which also take into account other operations such as spatial and moving object operations.

\section{Conclusions}

Modeling the future movements of moving objects has always been a complex task due to the inherent uncertainty aspect of the future. A few existing models have tried to solve the problem by selecting a specific type of movement, defining a specific prediction model, predicting a specific future movement, and answering queries about the prediction. In this paper, we have emphasized the necessity of separating an object model from a prediction model which leads us to our main contribution, an abstract model for all possible future movements of moving objects in general. Although this abstract model already offers many interesting elements, as a future work, we plan to extend this model further to better serve as a conceptual basis for finite representation models of future evolutions of moving objects. We also plan to investigate the integration of FuMMO with our historical movement model of moving objects in $[2]$. 


\section{References}

1. Pelekis, N., Theodoulidis, B., Kopanakis, I., Theodoridis, Y.: Literature Review of Spatio-Temporal Database Models. Knowledge Engineering Review (2005)

2. Güting, R., Böhlen, M., Erwig, M., Jensen, C., Lorentzos, N., Schneider, M., M.Vazirgiannis: A Foundation for Representing and Querying Moving Objects. ACM Trans. on Database Systems (TODS) 25(1) (2000) 881-901

3. Tossebro, E., Güting, R.: Creating Representations for Continuously Moving Regions from Observations. In: Int. Symp. on Advances in Spatial and Temporal Databases. (2001) 321-344

4. Erwig, M., Güting, R., Schneider, M., Vazirgiannis, M.: Abstract and Discrete Modeling of Spatio-Temporal Data Types. In: ACM Symp. on Geographic Information Systems (ACM GIS). (1998,) 131-136

5. Forlizzi, L., Güting, R., Nardelli, E., Schneider, M.: A Data Model and Data Structures for Moving Objects Databases. In: ACM SIGMOD Int. Conf. on Management of Data. (2000,) 319-330

6. Erwig, M., Güting, R., Schneider, M., Vazirgiannis, M.: Spatio-Temporal Data Types: An Approach to Modeling and Querying Moving Objects in Databases. Geoinformatica Journal 3(3) (1999) 265-291

7. Cotelo Lema, J.A., Forlizzi, L., Güting, R., Nardelli, E., Schneider, M.: Algorithms for Moving Objects Databases. The Computer Journal 46(6) (2003) $680-712$

8. Sistla, A., Wolfson, O., Chamberlain, S., Dao, S.: Modeling and Querying Moving Objects. In: Int. Conf. on Data Engineering (ICDE). (1997) 422-432

9. G. Trajcevski, O. Wolfson, K.H., Chamberlain, S.: Managing Uncertainty in Moving Objects Databases. ACM Trans. on Database Systems (TODS) 29 (2004) 463-507

10. Mokhtar, H., Su, J.: Universal Trajectory Queries for Moving Object Databases. In: Int. Conf. on Mobile Data Management (MDM), IEEE Computer Society (2004)

11. J. Chen, X. Meng, Y.G.S.G., Sun, H.: Modeling and Predicting Future Trajectories of Moving Objects in a Constrained Network. In: Int. Conf. on Mobile Data Management (MDM). (2006) 156 